\title{
Constraints on Higgs physics from EW precision measurements
}

\section{Satoshi Mishima*}

University of Rome "La Sapienza"

E-mail: Satoshi.Mishima@romal.infn.it

The electroweak precision measurements offer a very powerful handle on the underlying mechanism of electroweak symmetry breaking and on the nature of the Higgs boson. In this talk, I will present current constraints on new physics beyond the Standard Model from the electroweak precision fit, and discuss the interplay between the electroweak precision fit and the Higgs measurements at the Tevatron and LHC.

Frontiers of Fundamental Physics 14 - FFP14,

15-18 July 2014

Aix Marseille University (AMU) Saint-Charles Campus, Marseille

\footnotetext{
* Speaker.
} 